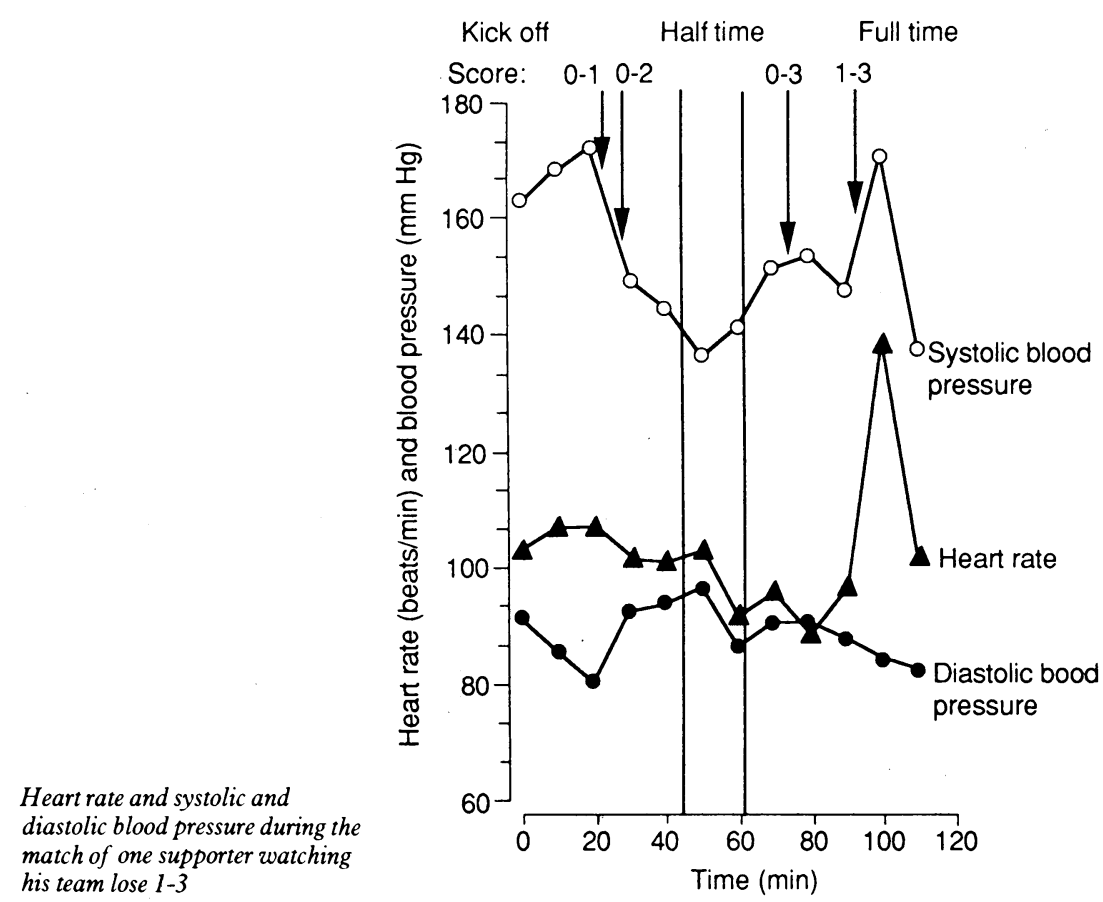

his team lose 1-3
Gloucester Royal Hospital, Gloucester GL1 3NN A J Makin, MRCP, senior house officer in general medicine

K A V Cartwright, FRCPATH, consultant microbiologist R A Banks, MD, consultant physician and nephrologist

Correspondence to:

Dr Banks.

BMF 1991;303:1610-1
$4,4,5,6,7$ and $7:$ seven men scored the match as 5 or less. There was a significant correlation between this subjective measure of excitement and the heart rate in the 30 seconds after the first goal was scored by the supported team (Spearman rank correlation coefficient $0.675 ; 0.05>p>0.01)$. There was, however, no correlation with any of the three variables measured over the whole match period, or with the change in their values between the home and match periods. The figure. shows the haemodynamic response of one fan to his team's 1-3 defeat.

\section{Discussion}

Although previous studies have shown the heart rate response to stressful daily activities such as car drivinges and occasional activities such as public speaking ${ }^{5}$ or $\overrightarrow{0}$ riding a roller coaster, ${ }^{6}$ few studies have additionally documented the effect of emotional stress on blood pressure beyond a controlled experimental environ-O ment.

We have shown a modest but significant increase in blood pressure (of a degree similar to that occurringe during light exercise) and heart rate in healthy meno watching football, most of whom rated the matches $\vec{v}$ studied as relatively unexciting.

Although these changes are not as great as those walking (median difference 13.0 (1 to 23 ) $\mathrm{mm} \mathrm{Hg}$; $0.05>p>0.02$, Wilcoxon signed rank test).

In all 10 men the mean heart rate in the 30 seconds after the supported team scored its first goal was higher than in the 30 seconds before the goal. Median heart rate for the whole group in the 30 seconds before a goal was scored was 96 beats/min (interquartile range $86-101)$; it was 120 beats/min (109-129) in the 30 seconds after; median difference +23 (16 to 30$)$ beats/min; $\mathrm{p}<0.01$, Wilcoxon signed rank test. All subjects recorded their maximum heart rate during the match in the 30 seconds following a goal for their team; in five this was the maximum rate recorded during the entire study period, the others having a higher rate recorded at least once during their journey to the match. The individual mean heart rate in the 30 seconds after the first goal for the supported team was a mean of $66 \%$ of the maximum predicted heart rate and in four men was greater than $70 \%$ of this value. No significant arrhythmia was documented in any subject.

The linear analogue excitement scores were $1,2,3,4$, found during other non-sporting activities such aso roller coaster riding, ${ }^{6}$ they were sustained over $\mathrm{a}^{\supset}$ longer period -90 minutes - and despite the subjective opinions of the supporters in this study and the intermittent doubts expressed by some sections of the English media - such as London Weekend Tele-ב vision's Saint and Greavsie - they may provide somed evidence that Scottish football is indeed exciting.

I Schiffer F, Hartley LH, Schulman CL, Abelmann WH. The quiz electrocardiogram. Am $\mathcal{F}$ Cardiol 1976;37:41-7.

2 Taggert $P$, Gibbons D, Somerville $W$. Some effects of motor car driving on the normal and abnormal heart. BMF 1969;iv: 130-4.

3 Northcote RJ, MacFarlane PW, Ballantyne D. Ambulatory electrocardio graphy in squash players. Br Heart $\mathcal{f} 1983 ; 50: 372-7$.

4 Clark S, Fowlie S, Coats A, Radaelli A, van der Putt M, Bird R, et al Ambulatory blood pressure monitoring: validation of the accuracy and reliability of the TM-2420 according to the AAMI recommendations f Hum Hypertens 1991;5:7-82.

5 Taggert $P$, Carruthers $M$, Somerville $W$. Electrocardiogram, plasma catechola mines and lipids, and their modification by oxprenolol when speaking before? mines and lipids, and their modice

6 Pring SD, LacFarlane PW, Cobbe SM Response of heart rate to a rollo coaster ride. BMf 1989;299:1575.

\title{
Keeping the cat out of the bag: a hazard in continuous ambulatory peritoneal dialysis
}

\section{A J Makin, K A V Cartwright, R A Banks}

\section{Introduction}

Continuous ambulatory peritoneal dialysis (CAPD) is an established method of renal replacement therapy. It uses the patient's own peritoneum as a dialysis membrane by running fluid from a bag into the abdominal cavity through a closed system consisting of a silastic catheter and a plastic dialysis line. The fluid is retained in the abdomen for several hours' "indwell" and is then drained out. This process is repeated several times a day. We report an unrecognised hazard for patients receiving this treatment.

\section{Case reports}

Case 1-A 39 year old man who had been receiving continuous ambulatory peritoneal dialysis for one year presented an hour after his kitten had bitten through his dialysis line while fluid was running into his abdomen. He was given a single dose of intravenous vancomycin as prophylaxis and discharged. Eight hours later he was readmitted with severe peritonitis? $\mathrm{He}$ was feverish and had bilateral digital ischaemia, a rigid abdomen, and an ileus. He was given benzyl penicillin and peritoneal lavage with vancomycin ${ }^{\text {s }}$ and gentamicin into each dialysis bag. Nasogastric aspiration and intravenous fluids were necessary untiP bowel activity returned. Gram negative intracellula diplococci were seen in the dialysis fluid and intrå요 venous ampicillin was started. Subsequent culture grew Neisseria pharyngis; this was resistant to vancomycin but sensitive to ampicillin and gentamicin. Theo patient returned to continuous ambulatory peritoneas dialysis and made a full recovery.

Case 2-A 58 year old woman was draining in when her kitten scratched and punctured her dialysis line. She was given vancomycin and did not develop peri- 


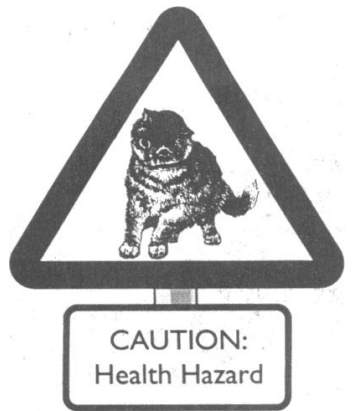

tonitis. Culture of the line grew Staphylococcus homini sensitive to vancomycin. The fluid was sterile.

Case 3-A 73 year old man had fluid indwell when his cat punctured the dialysis line with its claws. $\mathrm{He}$ was given vancomycin and had no further problems. The fluid was sterile.

Case 4-A 24 year old woman had her dialysis line bitten by her kitten while she was asleep with fluid indwell. She was given vancomycin and was started on oral ciprofloxacin. She remained free of symptoms; the fluid was sterile.

\section{Comment}

The nature of continuous ambulatory peritoneal dialysis, with a patient using four exchanges a day and having to break the closed system 1500 times per year, means that peritonitis is a common problem-it has an incidence of 1.5 episodes per patient per year. ${ }^{1}$ The most common source of infection is the patient's own skin flora, Staphylococcus epidermis, accounting for $53 \%$ of culture positive episodes. ${ }^{1}$ Antistaphylococcal drugs are therefore given as soon as possible after an accidental breach of the closed system.

We are not aware of previous reports of pets causing peritonitis by puncturing the closed system, but poor hygiene after disposal of poodle faeces has been reported to have resulted in peritonitis. ${ }^{2}$ Of the 10 line breaks in our CAPD programme in two years, four have been caused by cats.

A further problem when animals are involved, which is highlighted in our first case, is that the resulting peritonitis may be caused by an organism not sensitive to firstline antibiotics. Peritonitis from Neisseria species without animal interference has been described, ${ }^{34}$ but it is rare and probably results from haematogenous spread from the patient's oropharynx. ${ }^{4}$ Neisseria species have been isolated from the supragingival plaque of domestic cats. ${ }^{5}$ This suggests that the peritonitis in our first case resulted from direct inoculation of the organism into the fluid as it was running into the abdomen.

We conclude that cats are a significant hazard for patients receiving continuous ambulatory peritoneal dialysis, and we recommend some precautions for patients with a cat. The animal must not be in the same room when the patient is performing a fluid exchange; the animal must not sleep in the bedroom with the patient at night; dialysis fluid must be stored away from pets. Finally, interference by a cat may result in an infection with an unusual organism and treatment should be adjusted accordingly.

1 Heaton A, Rodger RSC, Sellars L, Goodship THJ, Fletcher R, Nikolakakis N e al. Continuous ambulatory peritoneal dialysis after the honeymoon: review of experience in Newcastle 1979-84. BMF 1986;293:938-41.

2 Fannelli C, Peterson CJ, Schoolworth AC. Poodle peritonitis. Peritoneal Dialysis International 1990;10:247-8.

3 Dadone C, Vigano F, Mariani P, Giltri C. Peritonitis caused by Neisseria species in CAPD. Peritoneal Dialysis Bulletin 1985;5:138.

4 Damani NN, Chin ATL, Branhamella catarrhalis peritonitis in a continuous ambulatory peritoneal dialysis patient. Nephron 1987;45:160-1.

5 Dent VE. Identification of oral Neisseria species of animals. 3 Appl Bacterio 1982;52:21-30.

\section{A potpourri of parasites in poetry and proverb}

\section{A Burns}

Ectoparasites, you may consider, hardly provide suitable material for flights of poetic fancy, but it is surprising how many verses have been composed with fleas, lice, and scabies as the main subject matter. Many of these express sentiments inclined towards affection for these cutaneous passengers, and a few even mention parasites in an amorous setting. I have not been able to discover any poetic works of note relating to the crab louse, most of the references to this louse having been committed to the walls of public conveniences by contemplative graffiti artists, and thereby eventually lost to scrutiny under successive layers of emulsion paint. It is a pity that this much maligned louse has not, as far as I am aware, attracted the attention of a poet whose creativity stretches beyond "There was a young lady from Hitchin...."

\section{Scabies mites}

As we all know, scabies is transmitted by close physical contact, and not by contact with fomites. But it was not until the work of Mellanby that the necessity for close physical contact in the spread of scabies was appreciated..$^{12} \mathrm{He}$ conducted a number of experiments using volunteers in the early 1940 s and established that clothing and bedding were not important in the spread of scabies. He also realised that there is a latent period of about four weeks after infection is acquired before itching begins. His volunteers were a group of conscientious objectors, and the research establishment was a house in the Sheffield suburb of Broomhill. The group produced its own coat of arms-bearing the motto "Itch dien."

At one stage in Mellanby's investigations the findings of his research were included in a play in verse about scabies and its social implications. The play was produced at a Christmas party and contained this offering:

Recondite research on Sarcoptes

Has revealed that infections begin

At home with your wives and your children

Or when you are living in sin.

Except in the case of the clergy

Who accomplish remarkable feats

And catch scabies and crabs

From doorhandles and cabs

And from blankets and lavatory seats.

Scabies mites burrow in the epidermis, and this activity is celebrated in one stanza of a delightful poem originating from Guy's Hospital:

There's a squeak of pure delight from a matey little mite,

As it tortuously tunnels in the skin.

Singing furrow, Folly furrow, come and join me in my burrow,

And we'll view the epidermis from within. ${ }^{3}$

Many remedies for scabies have been suggested, but for centuries the standard treatment was with sulphur. In fact, sulphur ointment is still used by some dermatologists. Rotenone, an extract of derris root, was used in the 1940s to treat scabies. Although it was effective, it was rather irritant, particularly to the delicate body parts of the male. In 1937 Kissmeyer introduced benzyl benzoate as a scabicide, and it has remained in use ever since. These three therapeutic agents are celebrated in this offering from the 1940s:

The treatment most in vogue of late

Concerns the benzyl benzoate,

Made up in water it's applied

All over every patient's hide.

It cures a very high proportion

When used with care and skill and caution. 